\title{
Hybrid Heuristics for the Probabilistic Maximal Covering Location-Allocation Problem
}

\author{
Francisco de Assis Corrêa, Antonio Augusto Chaves, Luiz Antonio Nogueira \\ Lorena \\ LAC - Computer and Applied Mathematics Laboratory \\ INPE - Brazilian Space Research Institute \\ 12227-010 São José dos Campos - SP, Brazil. \\ e-mail address: lorena@lac.inpe.br fax number: +55-012-39456375 (L.A.N. Lorena).
}

\begin{abstract}
The Maximal Covering Location Problem (MCLP) maximizes the population that has a facility within a maximum travel distance or time. Numerous extensions have been proposed to enhance its applicability, like the probabilistic model for the maximum covering locationallocation with constraint in waiting time or queue length for congested systems, with one or more servers per service center. This paper presents one solution procedure for that probabilistic model, considering one server per center, using a Hybrid Heuristic known as Clustering Search (CS), that consists of detecting promising search areas based on clustering. The computational tests provide results for network instances with up to 818 vertices.
\end{abstract}

Keywords: Location problems, covering problems, congested systems, clustering search.

\section{Introduction}

The Maximal Covering Location Problem (MCLP) has been extensively studied in the literature since its formularization by [Church and ReVelle (1974)]. The main objective of the MCLP is to choose the location of facilities to maximize the population that has a facility within a maximum travel distance (or time). Thus, a population is considered covered if it is within a predefined service distance (or time) of at least one of the existing facilities. The MCLP does not require that all demand areas be covered, but offers service to the maximum population, considering the available resources.

Since its proposal, numerous extensions of the MCLP have been proposed to enhance its applicability, both in public and private sectors. Applications range from emergency services [Eaton et. al. (1986)] [Current and O'Kelly (1992)], hierarchical health [Moore and ReVelle (1982)], air pollution control [Hougland and Stephens (1976)], to congested systems [Marianov and Serra (1998)] [Marianov and Serra (2001)]. Solutions methods for the MCLP include linear programming relaxation 
[Church and ReVelle (1974)], greedy heuristics [Daskin (1995)], Lagrangean Relaxation [Galvão and ReVelle (1996)], the Lagrangean/Surrogate heuristic [Lorena and Pereira (2002)], column generation [Pereira, Lorena and Senne (2007)]. Considerable revision of this subject can be found in [Chung (1986)], [Hale and Moberg (2003)], [Serra and Marianov (2004)] and [Galvão (2004)].

In many studies involving location problems, the distance (or time) between demand points and the facilities to which they have been allocated is the factor that represents the quality of the services that are given to the users. However, when service networks are projected, as in health systems or banking, the location of service centers has a strong influence on the congestion of each of them, and, consequently, the quality of services must be better defined and not only considering the travel distance or time. The centers must be located to allow the users to arrive at the center in an acceptable time, but it is desirable that the waiting time for service be no longer than a given time limit or that nobody stands in line with more than a predetermined number of other clients. These are important parameters in the measure of the desired quality [Marianov and Serra (1998)].

Congestion happens when a service center is not able to deal, simultaneously, with all the service requests that are made to it. Normally, the traditional models that deal with congestion include a capacity constraint, which forces the demand for service, normally constant in time and equal to an average, to be smaller than the maximum capacity of the center all the time. This is a deterministic approach to the problem, not considering the dynamic nature of the congestion. Depending on how the capacity constraint is developed, this means that the solution model produces idle servers, or results in a system that is not able to deal with all the demand [Marianov and Serra (1998) (2001)].

[Marianov and Serra (1998)] have proposed models based on the fact that the number of requests for services are not constant in time, but a stochastic process whose stochasticity of demand is explicitly considered in the capacity constraints. Instead of being limited to a maximum value, the authors define a minimum limit for the quality of the service reflected in the waiting time or the number of people waiting for service. The authors have developed the Queuing Maximal Covering LocationAllocation Model (QM-CLAM). Good reviews of the probabilistic models can be found in [Galvão (2004)] and [Brotcorne, Laporte and Semet (2003)].

[Correa and Lorena (2006)] have applied the Constructive Genetic Algorithm (CGA) in the QM-CLAM. The CGA works with a population formed by schemata (incomplete solutions) and structures (the complete solution), with the traditional operators: selection, recombination and mutation. The CGA differs from a classical GA in the way it evaluates the schemata, in its capacity to use heuristics to define the fitness evaluation function, and in its treatment of a dynamic population. A dynamic 
population is initially formed only by schemata, but may be enlarged after the use of recombination operators, or made smaller along the generations, guided by an evolutionary parameter. The dynamic population is built, generation after generation, by directly searching for well-adapted structures (a complete solution) and also for good schemata [(Lorena and Furtado (2001)] [Oliveira and Lorena (2004)] [Oliveira and Lorena (2005)].

The purpose of this paper is to examine the QM-CLAM with one server per service center and present a solution using a hybrid heuristic called Clustering Search (CS), which was proposed by [Oliveira and Lorena (2004) (2007)]. The CS consists of detecting promising areas of the search space, using an algorithm that generates solutions to be clustered. These promising areas may then be explored through local search methods as soon as they are discovered. The CS results are compared with those obtained by CGA and by the heuristic proposed by [Marianov and Serra (1998)].

The commercial solver CPLEX [ILOG (2006)] has been used to approximately solve the formulation for all problems, in order to validate the computational results of CS.

The remainder of the paper is organized as follows. Section 2 presents a mathematical formulation for QM-CLAM. Section 3 describes the heuristic that was used in this paper, and section 4 present the CS applied to QM-CLAM. Section 5 presents the computational results and section 6 concludes the paper.

\section{QM-CLAM}

The traditional Maximum Covering Location Problem (MCLP) proposed by [Church and ReVelle (1974)] cannot be used to deal with the congestion constraints, because there are no allocation variables. So, it is impossible to compute the requests for services that arrive at a center, and, consequently, to determine when congestion occurs. Thus, the MCLP has been rewritten as a p-median-like model, modified to accommodate the location and allocation variables. The objective is to maximize the covered population, considering a predefined number of service centers $(p)$.

An integer linear programming formulation for the QM-CLAM is obtained by introducing the following variables. Let $y_{j}=1$ if a center is located at a node $j$ and $y_{j}$ $=0$ otherwise; $x_{i j}=1$ if the users located at demand node $i$ are allocated to a center located at $j$, and $\mathrm{x}_{i j}=0$ otherwise. We consider $i \in I$ and $j \in N_{i}$, such that $I$ is a set of demand nodes, and $N_{i}$ is either a set of candidate locations that are within a standard distance from node $i$, or the set of candidate locations which can be reached from node $i$ within a certain standard time. Let $a_{i}$ be the total population at demand node $i$. The formulation of the model is [Marianov and Serra (1998)]: 


$$
\mathrm{v}(\mathrm{QM}-\mathrm{CLAM})=\operatorname{Max} \sum_{i, j} a_{i} x_{i j}
$$

Subject to

$$
\begin{gathered}
x_{i j} \leq y_{j} \quad i \in I, \quad j \in N_{i} \\
\sum_{j \in N_{i}} x_{i j} \leq 1 \quad i \in I \\
\text { or }\left\{\begin{array}{c}
\mathrm{P}(\text { center } j \text { has } \leq \text { b people in queue }) \geq \varphi \\
\mathrm{P}(\text { waiting time at center } j \leq \tau) \geq \varphi \\
\sum_{i \in I} y_{i}=p \\
y_{j}, x_{i j} \in\{0,1\}, \quad i \in I, \quad j \in N_{i}
\end{array}\right.
\end{gathered}
$$

The objective (1) maximizes the population allocated to a center. Constraint (2) defines that a demand point $i$ can be allocated to a node $j$ only if there is a center in $j$. Constraint (3) forces each demand node $i$ to be allocated to at most one service center $j$. Constraints (4) ensure that each center has no more than $b$ people on a line, with a probability of at least $\varphi$. Constraints (4a), make the total time spent by a user at a center $j$ be shorter than, or equal to $\tau$, with a probability of at least $\varphi$. Constraint (5) sets the number of centers to be located. Constraints (6) define the integrality requirements.

In order to write constraint (4), there is an assumption that requests for service at each demand node $i$ appear according to a Poisson process with intensity $f_{i}$. The service requests at a center are the union of the requests for service of the demand nodes, and they can be described as another stochastic process, equal to the sum of several Poisson processes, with intensity $\omega_{j}$ :

$$
\omega_{j}=\sum_{i \in I} f_{i} x_{i j}
$$

which means that, if the variable $x_{i j}$ is one, node $i$ is allocated to center $j$ and the corresponding intensity $f_{i}$ will be included in the computation of $\omega_{j}$. The well-known results for a $\mathrm{M} / \mathrm{M} / 1$ queuing system have been considered for each center and its allocated users [Larson and Odoni (1981)]. An exponentially distributed service time, with an average rate $\mu_{j}$, has been considered in those models, where $\mu_{j} \geq \omega_{j}$; otherwise, the system does not reach the equilibrium. 
The QM-CLAM can be formally stated as:

$$
\mathrm{v}(\mathrm{QM}-\mathrm{CLAM})=\operatorname{Max} \sum_{i, j} a_{i} x_{i j}
$$

Subject to

$$
\begin{gathered}
x_{i j} \leq y_{j} \quad i \in I, \quad j \in N_{i} \\
\sum_{j \in N_{i}} x_{i j} \leq 1 \quad i \in I \\
\text { or }\left\{\begin{array}{l}
\sum_{i \in I} f_{i} x_{i j} \leq \mu_{j} \sqrt[b+2]{1-\varphi} \quad j \in N_{i} \\
\sum_{i \in I} f_{i} x_{i j} \leq \mu_{j}+\frac{1}{\tau} \ln (1-\varphi) \quad j \in N_{i} \\
\sum_{i \in I} y_{i}=p \\
y_{j}, x_{i j} \in\{0,1\}, \quad i \in I, \quad j \in N_{i}
\end{array}\right.
\end{gathered}
$$

The complete calculations can be found in Marianov and Serra (1998). The QMCLAM belongs to the NP-Complete class of problem [Pirkul and Schilling (1991)]. Even when using commercial solvers, it is not always possible to find the optimal solution in a reasonable computational time, due to its classification and to the problem size. Therefore, alternative methods are investigated. In the next Section a solution based on the Clustering Search will be presented.

\section{Clustering Search Method}

The Clustering Search (CS), proposed by [Oliveira and Lorena (2004) (2007)], employs clustering for detecting promising areas of the search space. The sooner these strategic areas can be identified, the sooner a more accurate search strategy can be applied. An area can be seen as a search subspace defined by a neighborhood relationship in metaheuristic coding space. In the CS, a clustering process is executed simultaneously to a metaheuristic, identifying groups of solutions that deserve special attention.

The CS attempts to locate promising search areas by framing them by clusters. A cluster can be defined as a tuple $\mathrm{G}=\{\mathrm{c} ; \mathrm{r} ; \mathrm{s}\}$ where $c, r$ and $s$ are, respectively, the center and the radius of the area, and a search strategy associated to the cluster. 
The center of the cluster is a solution that represents the cluster, identifying its location inside the search space. Initially, the centers of the clusters are obtained randomly; but progressively, they tend to fall along really promising points in the close subspace. The radius $r$ establishes the maximum distance, starting from the center, for which a solution can be associated to the cluster. For example, in combinatorial optimization, $r$ can be defined as the number of movements needed to change a solution into another one. The search strategy is a systematic search intensification, in which solutions of a cluster interact among themselves along the clustering process, generating new solutions.

The CS consists of four conceptually independent components with different attributions:

- search metaheuristc (SM);

- iterative clustering (IC);

- analyzer module (AM);

- local searcher (LS);

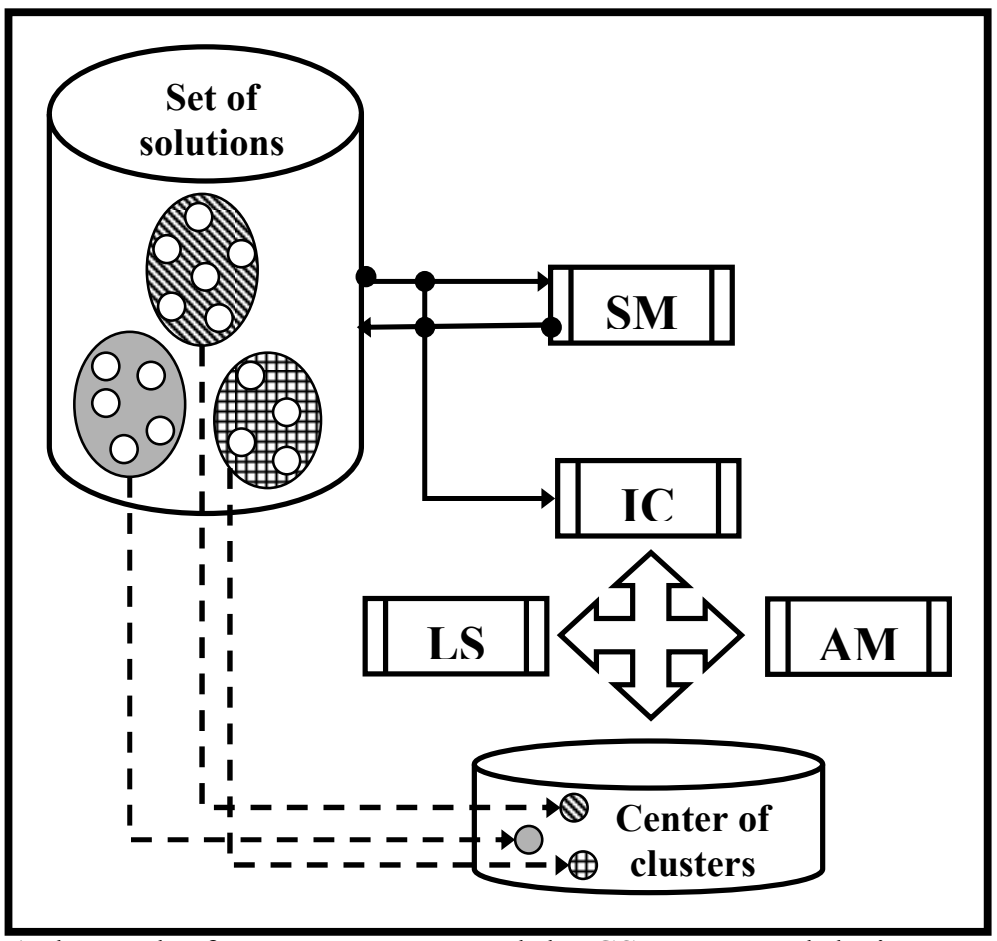

Figure 1 shows the four components and the CS conceptual design.

Figure 1. CS Components 
The SM works as a full-time solution generator. The algorithm is executed independently of the remaining components and must be able to provide a continuous generation of solutions to the clustering process. Clusters are maintained, simultaneously, to represent these solutions.

The IC aims to gather similar solutions into groups, identifying a representative cluster center for them. To avoid extra computational effort, IC is designed as an online process, in which the clustering is progressively fed by solutions generated in each iteration of SM. A maximum number of clusters NC is an upper bound value that prevents an unlimited cluster creation. A distance metric must be defined, a priori, allowing a similarity measure for the clustering process.

The AM provides an analysis of each cluster, at regular intervals, indicating a probable promising cluster. A cluster density, $\delta_{i}$, is a measure that indicates the activity level inside the cluster. For simplicity, $\delta_{i}$ counts the number of solutions generated by SM and allocated to the cluster $i$. Whenever $\delta_{i}$ reaches a certain threshold, indicating that some information template has become predominantly generated by SM, that information cluster must be better investigated to accelerate the convergence process on it.

Finally, the LS is a local search module that provides the exploitation of a supposed promising search area framed by a cluster. This process is executed each time AM finds a promising cluster. LS can be considered as the particular search strategy associated with the cluster, i.e., a problem-specific local search to be applied to the cluster.

\section{CS for QM-CLAM}

A version of CS for the QM-CLAM is presented in this section. A solution was represented through a computational structure that contains the located centers, the allocation of the demand points and the objective value. The allocation procedure tests all the service centers defined by the solution and chooses one to allocate each demand point that satisfies: a) the coverage; b) its capacity; c) the service center that will have the minimum accumulated demand.

The whole CS pseudo-code for the QM-CLAM is presented in Figure 2. The pseudocode shows where the different components of the procedure fit.

The component SM, responsible for generating solutions to the clustering process, was the metaheuristic Greedy Randomized Adaptive Search Procedure (GRASP) [Feo and Resende (1995)]. The GRASP is basically composed of two phases: a construction phase, in which a feasible solution is generated, and a local search phase, in which the constructed solution is improved. 


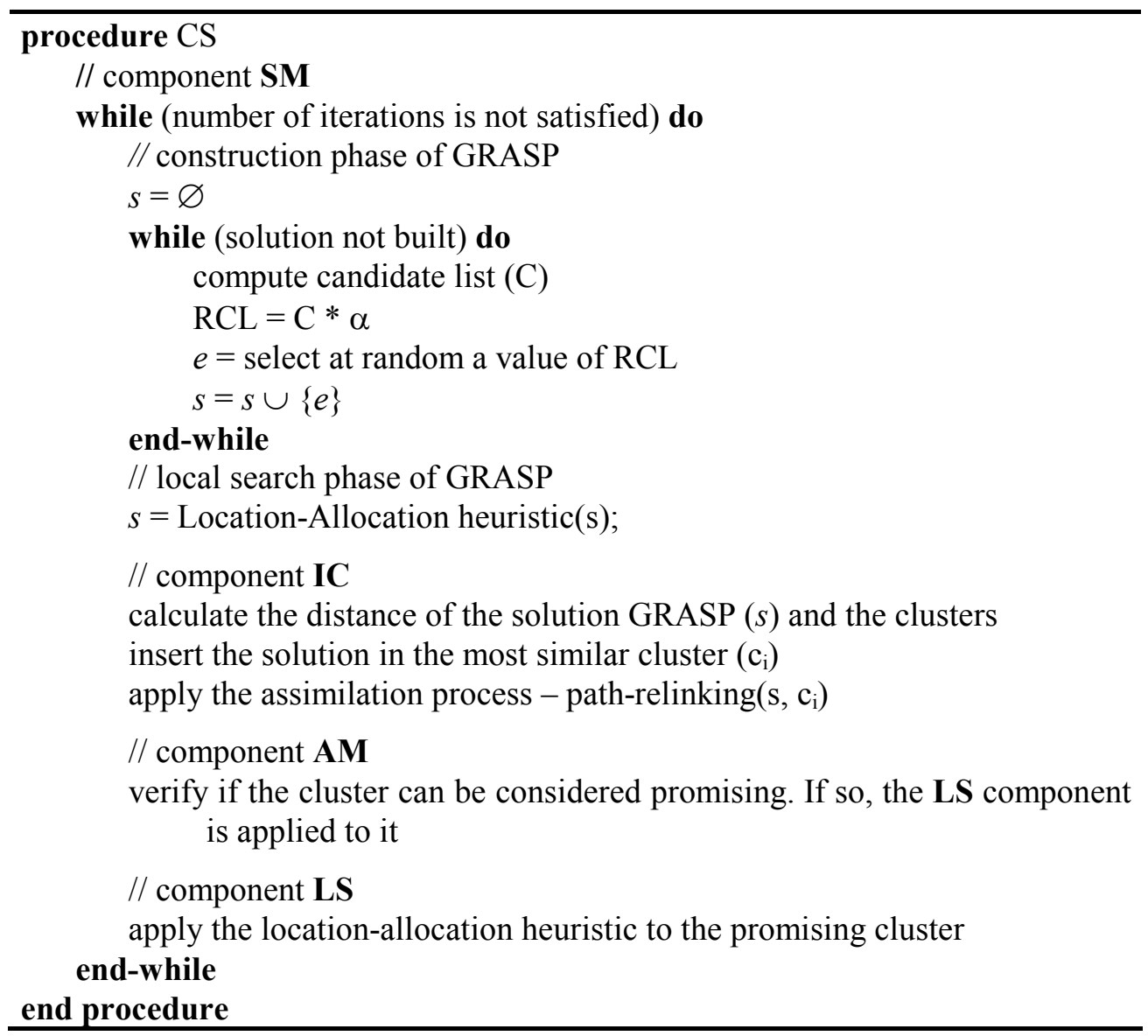

Figure 2. CS pseudo-code

The construction phase of GRASP uses the steps 1 to 5 of the greedy heuristic reported in [Marianov and Serra (1998)], but changes the way to locate a server in step 5. A candidate list (C) is built in step 1 with all candidate locations. A restrict candidate list (RCL) is built in step 5 with a percentage $\alpha$ of $C\left(R C L=C^{*} \alpha\right)$, with the highest current total incoming call rate. One node is chosen randomly in the RCL. Steps 1 to 5 used in this phase are shown in Figure 3.

The local search phase of GRASP uses the location-allocation heuristic, proposed by [Lorena and Pereira (2002)]. The solutions obtained from the construction phase can be improved by searching for a new center in each covered area, swapping the current center by a allocated node of the same covered area, and changing the allocation solution, as shown in Figure 4. The algorithm is shown in Figure 5. That change may alter both allocation and the coverage of the QM-CLAM solution; so it is necessary to recalculate allocation. 
The IC is the CS's core, working as a classifier, keeping in the system only relevant information, and guiding search intensification in the promising search areas. A maximum number of clusters $(N C)$ is defined a priori. The $i^{t h}$ cluster has its own center $c_{i}$ and a radius $r$, like the other clusters.

\section{Heuristic}

\section{Step 1:}

Make a list of the candidate locations $j$ (candidate list $\mathrm{C}$ ), ordered by a decreasing call rate (each demand node is a potential center location). Call this list $D_{j}$.

For each candidate location, compute the right-hand side of equation (4) or (4a), depending on the model being utilized. For both equations, the right-hand side is initially computed using all previously known values of $\mu, b$ and $\varphi$ (equation 4) or $\mu, b$ and $\tau$ (equation $4 a$ ).

For each candidate location $j$, make a list of all demand nodes $i$ within the standard distance, ordered by increasing distance to the candidate location. Call this list $D_{j i}$.

\section{Step 2:}

For each candidate location, make the current total incoming call rate equal zero $\left(\omega_{i n c, j}\right)$.

\section{Step 3:}

Starting with the first candidate location $j$ on the list $D_{j}$, add to its incoming call rate $\omega_{i n c, j}$, the call rate $f_{i}$ of the first node on the list $D_{j i}$. Then, add the call rate $f_{i}$ of the second node on the list $D_{j i}$, then the third, and so on, until the point where adding any extra demand node would exceed the limit value of calls $\omega_{\text {inc, }}$. Temporarily allocate all these demand nodes to a hypothetical service center at node $j$.

\section{Step 4:}

Repeat step 3 for all nodes in list $D_{j}$. Note that the same demand node could be temporarily allocated to several candidate locations.

\section{Step 5:}

Locate a service center on the node chosen randomly within a percentage $\alpha$ of the highest current total incoming call rates. Take all demand nodes allocated to it out of all the lists $D_{j i}$ of all potential centers. Allocate them definitively to the located center.

\section{Step 6:}

Repeat steps 2 to 5 until all available centers are located.

\section{End-Heuristic}

Figure 3. Heuristic used in the construction phase of GRASP 


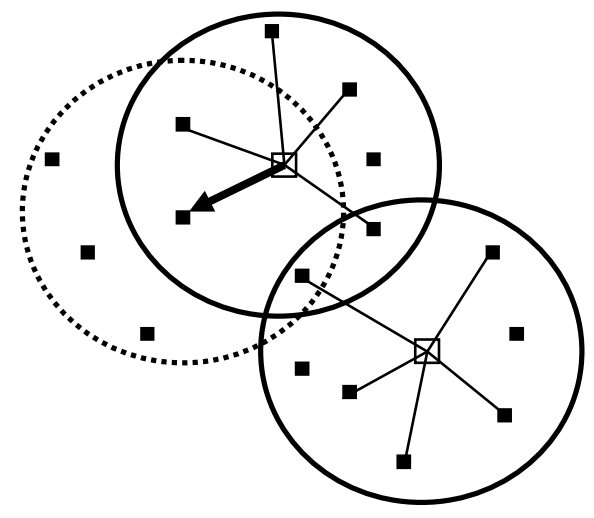

(a) Initial Solution

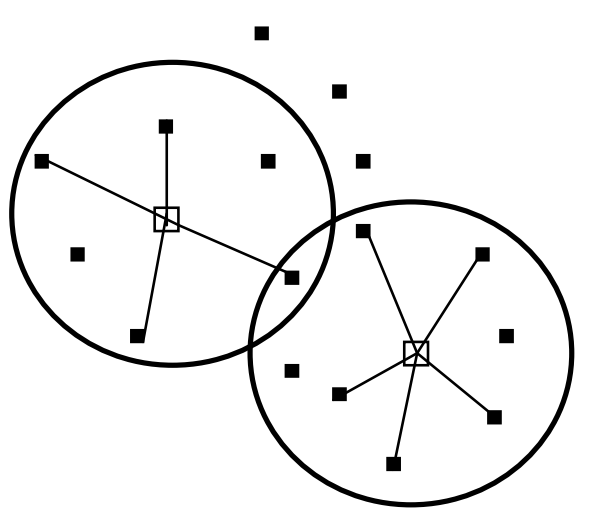

(b) After reallocation

Figure 4. Reallocation of nodes.

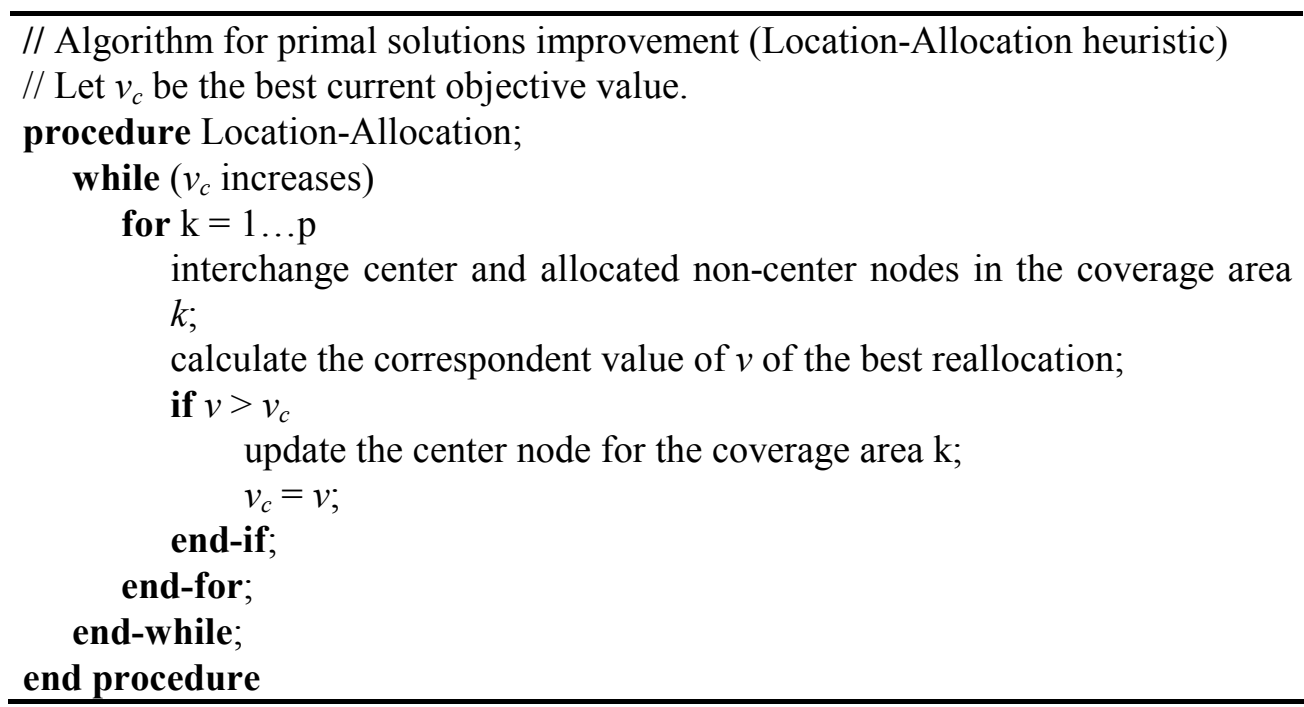

Figure 5. Primal solutions improvement

Solutions generated by GRASP (SM) are passed to IC that attempts to group these solutions as known information in a cluster, chosen according to a distance metric. The solution activates the closest center $c_{i}$ (cluster center that minimizes the distance metric), and a disturbance is applied to it. In this paper, the metric distance is the number of different located centers between the GRASP and the center of the cluster 
solutions. When there are a larger number of different located centers between the GRASP and the cluster solution center it increases the dissimilarity. The disturbance is an assimilation process, in which the center of the cluster is updated by the new generated solution. In this paper, this process is the path-relinking method [Glover (1996)], that generates several points (solutions) along the path that connects the solution generated by GRASP and the one in the center of the cluster. Since each point is evaluated by the objective function, the assimilation process itself is an intensification mechanism inside the clusters. The new center $c_{i}$ is the best-evaluated solution obtained in the path.

Path-relinking starts from two elite solutions. The first is the solution that comes from the SM component $\left(s_{\text {initial }}\right)$. The second is the closest cluster center $c_{i}\left(s_{\text {guide }}\right)$. The procedure starts by computing the symmetric difference between the two solutions $\Delta\left(s_{\text {initial }}, s_{\text {guide }}\right)$, i.e. the set of moves needed to reach $s_{\text {guide }}$ from $s_{\text {initial }}$. A path of solutions is generated, linking $s_{\text {initial }}$ and $s_{\text {guide }}$. At each step, the procedure examines all moves $m \in \Delta\left(s_{\text {initial }}, s_{\text {guide }}\right)$ from the current solution $s$ and selects the one which results in the best cost solution, applying the best move $\left(m^{*}\right)$ to solution $s\left(s \oplus m^{*}\right)$. The set of available moves is updated. The procedure terminates when $s_{\text {guide }}$ is reached, i.e. when $\Delta\left(s_{\text {initial }}, s_{\text {guide }}\right)=\varnothing$. The best solution $s^{*}$ in this path is returned by the algorithm. In this paper, one move is to swap a median of the $s_{\text {initial }}$ by a median of the $s_{\text {guide }}$, changing the allocation solution. Figure 6 illustrates the pseudo-code of the path-relinking and Figure 7 shows an example for a 30-node network with five service centers.

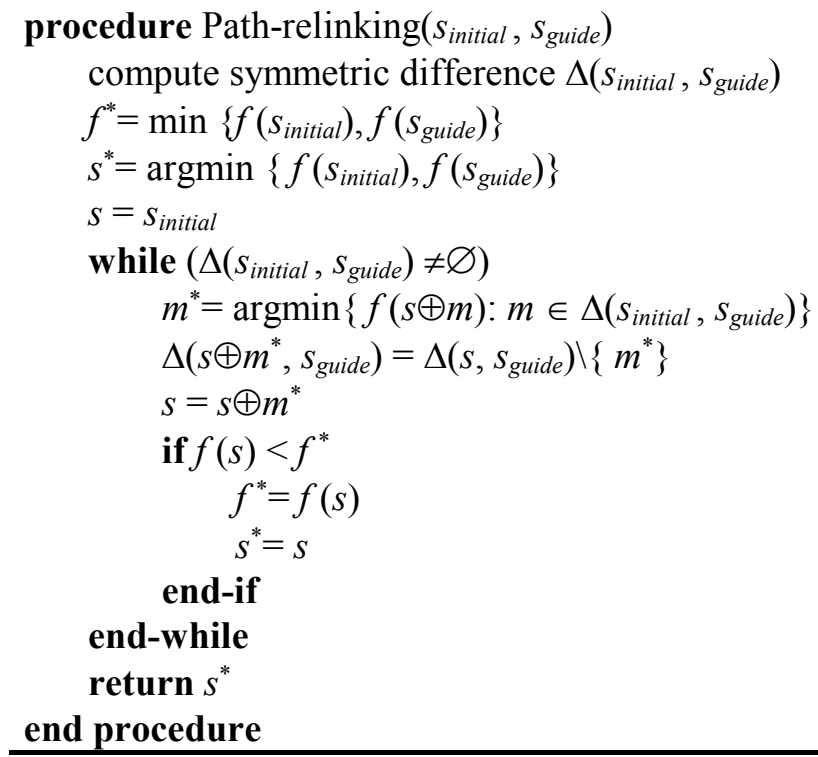


Figure 6. Path-relinking algorithm

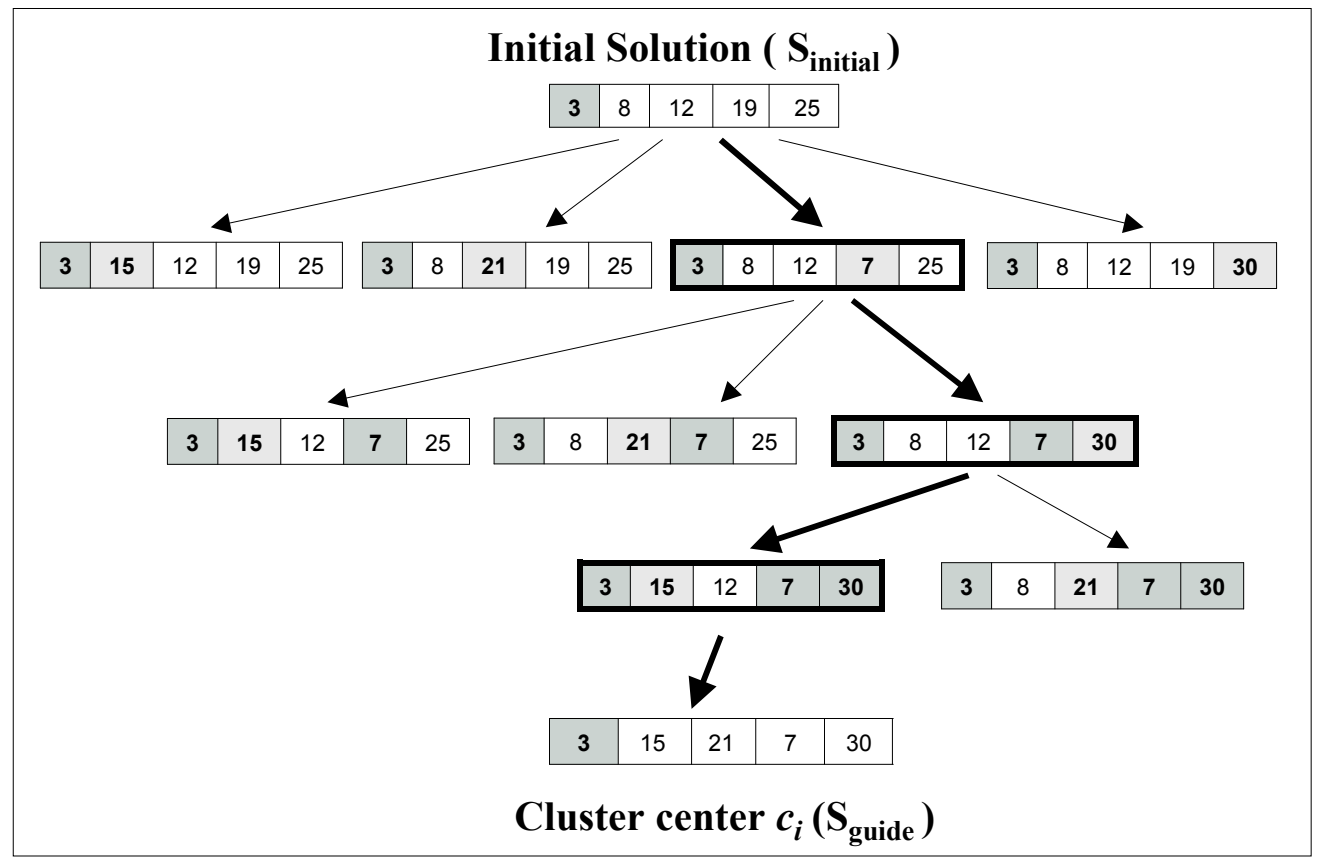

Figure 7. Path-relinking moves

The AM component is executed whenever a solution is assigned to a cluster, verifying if the cluster can be considered promising. A cluster becomes promising when it reaches a density $\lambda_{i}$,

$$
\lambda_{i} \geq P D \cdot \frac{N S}{\left|C_{t}\right|}
$$

where, NS is the number of solutions generated in the interval of analysis of the clusters, $\left|\mathrm{C}_{t}\right|$ is the number of clusters in the iteration $t$, and $P D$ is the desirable cluster density beyond the normal density, obtained if NS were equally divided to all clusters. The center of a promising cluster is improved through the LS. The AM also performs the cooling of all clusters, halving the $\lambda_{i}$ value.

The component LS is activated when the AM discovers a promising cluster. The LS implementation uses the idea behind the local search phase of GRASP, the locationallocation heuristic [Lorena and Pereira (2002)], changing the way of searching for a new service center. Rather than swapping the current center by an allocated node of the same covered area, it swaps the current center by all node in the same covered area, changing the allocation solution, recalculating the coverage, and taking the best 
solution found as the new one. It permits searching for good solutions in different search spaces. After all, the LS applies the Swap2 heuristic (SW2) to all located centers, examining all possible swaps of two allocated and two non allocated demand points. The best one is performed and the process is repeated until no improvements occur. Therefore, the location-allocation heuristic searches for the best center location, and the Swap2 searches for the best allocation inside the best location found. After applying both heuristics, the cluster center is updated if the new solution is better than the previous one.

\section{Computational Results}

The CS was tested in the 30-node network provided by [Marianov and Serra (1998)] and in 324-node and 818- node networks obtained from a geographical data base of São José dos Campos, Brazil. These two networks were increased by fictitious population for each demand point and they are available at http://www.lac.inpe.br/ lorena/instancias.html. The 30-node network is shown in Table 1.

By varying the $p, b, \mu, \varphi$ e $\tau$ parameters, various problems have been created. The results from CS have been compared to the results obtained using the commercial solver CPLEX, version 10.0 [ILOG (2006)], from the CGA [Correa and Lorena (2006)] and from the heuristic proposed by Marianov and Serra (1998).

For the implementation of the QM-CLAM, the service centers are primary health care centers, with one physician at each center. Each demand point is also a potential center location (candidate location), and the distances are Euclidean. For the 30-node network, the following parameters were adopted: covered distance equaled to 1.5 miles; average service time $(1 / \mu)$ was set at 20 minutes; call rates were set at 0.015 times the node population for the constrained queue length and 0.006 times the node population for the constrained waiting time, all defined in [Marianov and Serra (1998)].

For the 324-node and 818-node networks, the following parameters were used: average service time $(1 / \mu)$ was set at 15 minutes; call rates were set at 0.01 times the node population for both constrained queue length and constrained waiting time. For the 324-node network, covered distance equaled 250 meters, and for the 818-node, equaled 750 meters.

The problems were codified in the following way: number of points, number of centers, constraint type ( 0 for the constrained queue length and 1 for the constrained waiting time), number of people in line or waiting time, and probability. For example: 324_20_0_2_95, means there are 324 points, 20 centers, a constrained queue length, with a maximum of two people in line, with the probability of at least 95\%. The CS code was written in Object Pascal. The times in tables are shown in 
seconds and were determined in a Pentium IV $3 \mathrm{GHz}$ computer, with $1 \mathrm{~Gb}$ of RAM memory. The times for the attainment of the CPLEX solutions were limited to 3 hours (10800 seconds). The instances marked with one * define a stop in the execution due to an out of memory error.

Table 2 shows the results obtained for the 30-node network; Table 3 shows the results for the 324-node network and Table 4, for the 818-node network. The values of Gap CPLEX equal to zero mean that the optimum was reached.

The results for the heuristic defined in [Marianov and Serra (1998)] (MS_Heuristic) are shown in three columns: Solution, Time, and Deviation. The Dev column (Deviation) shows, in percentage, the relative error between the heuristic and CPLEX solutions. Its values are calculated by (CPLEX solution - MS_Heuristic solution)/(CPLEX solution).

The results for the CGA and CS reflect fifty executions of each problem and are shown in four columns: the best value found (Best column), the average value (Average column), the average time (Time column) and the deviation (Dev column), that reflects, in percentage, the relative error between the best solution for the CGA or CS and the CPLEX, and is calculated by (CPLEX solution - Best solution)/(CPLEX solution). Therefore, the negative values of the deviations indicate that the best solution for the CS was better than the CPLEX solution. The values in boldface show the best solutions found.

The following values of parameters for CS were adjusted through several executions and are also based in [Oliveira and Lorena (2004)]. The following parameters produced good results:

- number of solutions generated at each analysis of the clusters $N S=15$;

- maximum number of clusters $N C=5$;

- density pressure $P D=2$;

In $75 \%$ of the cases, the full CS procedure found objective values that were better than those found by the GRASP alone (SM component of CS) for the 324-node network and in about $92 \%$ for the 818 -node network. It shows that the other components of CS contribute substantially to improve the solution values.

As shown in Table 2, for the 30-node network, CS found the optimal value in about $65 \%$ of the problems, considering the average results, and in about $81 \%$, considering the best values, with better average time than CGA and CPLEX.

For the 324-node network, CPLEX found better results in 100\% of the tests. However, it had found serious problems in two instances due to out of memory error, and it did not find the optimal value for three problems in three hours of execution. The running times of the CS were very competitive compared to CPLEX, with good 
solution values. CS found better results than the heuristic by Marianov and Serra (1998) and CGA.

For the 818-node network, the running times of the CS were very competitive as compared to the CPLEX, with better solution values in about $30 \%$ of the problems. CPLEX did not find the optimal value in about $46 \%$ of the instances tested in three hours of execution, and in some of them there were gaps above $100 \%$. CS found the same optimal values as CPLEX with better computational times. CS also obtained better results than the heuristic by Marianov and Serra (1998) and CGA.

Table 1. 30-node network

\begin{tabular}{cccccccc}
\hline \hline Node & X & Y & Population & Node & X & Y & Population \\
\hline $\mathbf{1}$ & 3.2 & 3.1 & 710 & $\mathbf{1 6}$ & 3.0 & 5.1 & 110 \\
$\mathbf{2}$ & 2.9 & 3.2 & 620 & $\mathbf{1 7}$ & 1.9 & 4.7 & 100 \\
$\mathbf{3}$ & 2.7 & 3.6 & 560 & $\mathbf{1 8}$ & 1.7 & 3.3 & 100 \\
$\mathbf{4}$ & 2.9 & 2.9 & 390 & $\mathbf{1 9}$ & 2.2 & 4.0 & 90 \\
$\mathbf{5}$ & 3.2 & 2.9 & 350 & $\mathbf{2 0}$ & 2.5 & 1.4 & 90 \\
$\mathbf{6}$ & 2.6 & 2.5 & 210 & $\mathbf{2 1}$ & 2.9 & 1.2 & 90 \\
$\mathbf{7}$ & 2.4 & 3.3 & 200 & $\mathbf{2 2}$ & 2.4 & 4.8 & 80 \\
$\mathbf{8}$ & 3.0 & 3.5 & 190 & $\mathbf{2 3}$ & 1.7 & 4.2 & 80 \\
$\mathbf{9}$ & 2.9 & 2.7 & 170 & $\mathbf{2 4}$ & 6.0 & 2.6 & 80 \\
$\mathbf{1 0}$ & 2.9 & 2.1 & 170 & $\mathbf{2 5}$ & 1.9 & 2.1 & 80 \\
$\mathbf{1 1}$ & 3.3 & 2.8 & 160 & $\mathbf{2 6}$ & 1.0 & 3.2 & 70 \\
$\mathbf{1 2}$ & 1.7 & 5.3 & 150 & $\mathbf{2 7}$ & 3.4 & 5.6 & 60 \\
$\mathbf{1 3}$ & 3.4 & 3.0 & 140 & $\mathbf{2 8}$ & 1.2 & 4.7 & 60 \\
$\mathbf{1 4}$ & 2.5 & 6.0 & 120 & $\mathbf{2 9}$ & 1.9 & 3.8 & 60 \\
$\mathbf{1 5}$ & 2.1 & 2.8 & 120 & $\mathbf{3 0}$ & 2.7 & 4.1 & 60 \\
\hline
\end{tabular}

\section{Conclusions}

This paper has presented a solution for the Probabilistic Maximal Covering LocationAllocation Problem using Clustering Search (CS). The CS is a new method that has been applied with success in some combinatorial optimization problems, such as the pattern sequencing problem [(Oliveira and Lorena (2004)] and prize collecting traveling salesman problem [Chaves and Lorena (2005].

The idea of the CS is to avoid applying a local search heuristic to all solutions generated by a metaheuristic, which can make the search process impracticable since it is time consuming, mainly when the heuristic has a high computational cost. The CS detects the promising regions in the search space during solution generation process, i.e., to detect promising regions becomes an interesting alternative in order to prevent the indiscriminate application of such heuristics. 
This paper reports results of different methods applied to QM-CLAM. CS got better results than others heuristics (CGA and the heuristic by Marianov and Serra) and it founds good values comparing to CPLEX. CS has two advantages over CPLEX: execution time, and the cost of a commercial solver.

The results show that the CS approach is competitive for the resolution of this problem in reasonable computational times. For some instances of 30-node and 818node networks, the optimal values were found. Therefore, these results validate the CS application to the QM-CLAM.

Further studies can be done which analyze others metaheuristics to generate solutions for the clustering process of CS, such as the Ant Colony System, Tabu Search and Genetic Algorithm, and which implement new local search heuristics for the QMCLAM. Moreover, larger instances of this model can be generated and solved. 
Table 2. Results for the 30-node network

\begin{tabular}{|c|c|c|c|c|c|c|c|c|c|c|c|c|c|c|c|c|c|}
\hline \multirow[t]{2}{*}{ Problem } & \multicolumn{3}{|c|}{ CPLEX } & \multicolumn{4}{|c|}{ CGA } & \multicolumn{3}{|c|}{ MS_Heuristic } & \multicolumn{3}{|c|}{ GRASP } & \multicolumn{4}{|c|}{ CS } \\
\hline & Sol. & Gap & Time & Best & Average & Time & Dev. & Sol. & Time & Dev. & Best & Average & Time & Best & Average & Time & Dev. \\
\hline 30_2_0_0_85 & 3700 & 0 & 0.22 & 3700 & 3700 & 0.31 & 0.00 & 3700 & 0.00 & 0.00 & 3700 & 3700.0 & 0.007 & 3700 & 3700.0 & 0.009 & 0.00 \\
\hline 30_3_0_0_85 & 5390 & 0 & 0.12 & 5390 & 5390 & 0.51 & 0.00 & 5210 & 0.00 & 3.34 & 5390 & 5390.0 & 0.019 & 5390 & 5390.0 & 0.025 & 0.00 \\
\hline 30_2_0_1_85 & 5100 & 0 & 3.31 & 5090 & 5090 & 0.36 & 0.20 & 4630 & 0.00 & 9.22 & 5090 & 5090.0 & 0.016 & 5090 & 5090.0 & 0.018 & 0.20 \\
\hline 30_3_0_1_85 & 5390 & 0 & 0.08 & 5390 & 5390 & 0.48 & 0.00 & 5210 & 0.00 & 3.34 & 5390 & 5390.0 & 0.021 & 5390 & 5390.0 & 0.024 & 0.00 \\
\hline 30_2_0_2_85 & 5210 & 0 & 0.20 & 5210 & 5210 & 0.38 & 0.00 & 4780 & 0.00 & 8.25 & 5210 & 5210.0 & 0.015 & 5210 & 5210.0 & 0.016 & 0.00 \\
\hline 30_3_0_2_85 & 5390 & 0 & 0.12 & 5390 & 5390 & 0.47 & 0.00 & 5210 & 0.00 & 3.34 & 5390 & 5390.0 & 0.022 & 5390 & 5390.0 & 0.023 & 0.00 \\
\hline 30_5_0_0_95 & 5330 & 0.38 & 10800 & 5330 & 5323 & 0.80 & 0.00 & 5210 & 0.00 & 2.25 & 5330 & 5312.8 & 0.032 & 5330 & 5317.6 & 0.041 & 0.00 \\
\hline 30_6_0_0_95 & 5410 & 1.11 & 10800 & 5410 & & 0.84 & 0.00 & 5390 & 0.00 & 0.37 & 5390 & 5390.0 & 0.029 & 5410 & & 0.037 & 0.00 \\
\hline 30_3_0_1_95 & 5270 & 0 & 116.00 & 5240 & & & 0.57 & 5080 & 0.00 & 3.61 & 240 & 5239.8 & 0.022 & 5240 & & 0.024 & .57 \\
\hline 30_4_0_1_95 & 5390 & 0 & 3.77 & 5390 & 5390 & 0.54 & 0.00 & 5260 & 0.00 & 2.41 & 5390 & 5390.0 & 0.022 & 5390 & 5390.0 & 0.026 & 0.00 \\
\hline 30_2_0_2_95 & 4520 & 0 & 2.11 & 4520 & 4513 & 0.30 & 0.00 & 4470 & 0.00 & 1.11 & 4520 & 4520.0 & 0.013 & 4520 & 4520.0 & 0.015 & 0.00 \\
\hline 30_3_0_2_95 & 5390 & 0 & 0.12 & 5390 & 5390 & 0.50 & 0.00 & 5230 & 0.00 & 2.97 & 5390 & 5390.0 & 0.023 & 5390 & 0.0 & 0.027 & 0.00 \\
\hline 30_4_1_48_90 & 1920 & 0 & 0.41 & 1920 & & 0.66 & 0.00 & 1890 & 0.00 & 1.56 & 1920 & & & 1920 & & 0.014 & 0.00 \\
\hline 30_5_1_48_90 & 2400 & 0 & 0.41 & 2390 & 2390 & 0.74 & 0.42 & 2280 & 0.00 & 5.00 & 2400 & 2398.8 & 0.013 & 2400 & 2398.8 & 0.019 & 0.00 \\
\hline 30_3_1_49_90 & 2160 & 0 & 0.31 & 2160 & 2160 & 0.53 & 0.00 & 2160 & 0.00 & 0.00 & 2160 & 2160.0 & 0.007 & 2160 & 2160.0 & 0.009 & 0.00 \\
\hline 30_4_1_49_90 & 2880 & 0 & 0.41 & 2880 & 2877 & 0.59 & 0.00 & 2870 & 0.00 & 0.35 & 2880 & 2880.0 & 0.008 & 2880 & 2880.0 & 0.013 & 0.00 \\
\hline 30_5_1_50_90 & 4700 & 0 & 4.09 & 4700 & 4700 & 0.73 & 0.00 & 4670 & 0.00 & 0.64 & 4700 & & 0.018 & 4700 & & 0.028 & 0.00 \\
\hline 30_6_1_50_90 & 5390 & 0 & 350.52 & 5390 & 5390 & 0.97 & 0.00 & 5060 & 0.00 & 6.12 & 5390 & 5390.0 & 0.027 & 5390 & 5390.0 & 0.038 & 0.00 \\
\hline 30_5_1_40_85 & 3050 & 0 & 3.66 & 3020 & 3001 & 0.74 & 0.98 & 2910 & 0.00 & 4.59 & 3050 & 3033.2 & 0.016 & 3050 & 3033.2 & 0.021 & 0.00 \\
\hline 30_6_1_40_85 & 3610 & 0 & 31.62 & 3610 & 3610 & 0.91 & 0.00 & 3480 & 0.00 & 3.60 & 3610 & 3606.8 & 0.021 & 3610 & 3608.8 & 0.031 & 0.00 \\
\hline 30_7_1_40_85 & 4060 & 0 & 16.89 & 4060 & 4060 & 1.03 & 0.00 & 3860 & 0.00 & 4.93 & 4060 & 4060.0 & 0.028 & 4060 & 4060.0 & 0.039 & 0.00 \\
\hline 30_6_1_41_85 & 5330 & 0.19 & 10800 & 5300 & 5274 & 1.00 & 0.56 & 5120 & 0.00 & 3.94 & 5270 & 5270.0 & 0.028 & 5330 & 5286.4 & 0.038 & 0.00 \\
\hline 30_7_1_41_85 & 5410 & 0 & 43.56 & 5390 & 5390 & 0.88 & 0.37 & 5300 & 0.00 & 2.03 & 5390 & 5390.0 & 0.023 & 5390 & 5390.0 & 0.035 & 0.37 \\
\hline 30_8_1_41_85 & 5470 & 0 & 0.05 & 5470 & 5470 & 0.95 & 0.00 & 5390 & 0.00 & 1.46 & 5470 & 5470.0 & 0.019 & 5470 & 5470.0 & 0.032 & 0.00 \\
\hline 30_4_1_42_85 & 4600 & 0 & 0.75 & 4600 & 4600 & 0.61 & 0.00 & 4550 & 0.00 & 1.09 & 4600 & 4600.0 & 0.016 & 4600 & 4600.0 & 0.022 & 0.00 \\
\hline 30_5_1_42_85 & 5390 & 0 & 6.50 & 5390 & 5390 & 0.77 & 0.00 & 5210 & 0.00 & 3.34 & 5390 & 5390.0 & 0.027 & 5390 & 5390.0 & 0.035 & 0.00 \\
\hline
\end{tabular}


Table 3. Results for the 324-node network

\begin{tabular}{|c|c|c|c|c|c|c|c|c|c|c|c|c|c|c|c|c|c|}
\hline Problem & \multicolumn{3}{|c|}{ CPLEX } & \multicolumn{4}{|c|}{ CGA } & \multicolumn{3}{|c|}{ MS_Heuristic } & \multicolumn{3}{|c|}{ GRASP } & \multicolumn{4}{|c|}{ CS } \\
\hline & & 0 & & & & 3.63 & 0.14 & & 0.14 & 0.34 & & & & 1455 & & 16 & 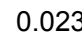 \\
\hline & & 0 & 305.3 & & & 7.81 & 0.09 & & 0.20 & 0.20 & & & 2.01 & & 45354.6 & 3.10 & 0.035 \\
\hline & 37180 & 0 & 481.0 & 7145 & 37069.0 & 8.10 & 0.09 & 37081 & 0.22 & 0.27 & 37157 & 37155.8 & 2.24 & 7173 & 7163.2 & 3.46 & 0.019 \\
\hline & & 0 & 92.1 & & & 3.54 & 0.09 & & & 0.42 & & & 2.18 & & & 3.37 & 0.007 \\
\hline & 293 & 0 & 216.5 & & & 8.27 & 0.12 & & & 0.25 & & & 2.23 & & 1.9 & 52 & 031 \\
\hline $2 \_85$ & 30950 & 0 & 1074.1 & 30932 & 95.0 & 8.34 & 0.06 & 30902 & 0.17 & 0.16 & 30928 & & 2.21 & 30948 & 3.3 & 3.33 & 0.006 \\
\hline 8_90 & 26920 & 0 & 421.6 & 26883 & 26835.0 & 8.35 & 0.14 & 26855 & 0.14 & 0.24 & 26899 & 396.6 & 2.18 & 26917 & 10.6 & 3.50 & 0.011 \\
\hline $9 \_90$ & 8330 & 0 & 9.1 & 8280 & & 8.28 & 0.18 & & 0.2 & 0.44 & 28295 & & 2.24 & & 0.3 & 3.43 & 0.042 \\
\hline 324 & 74315 & $0.061^{*}$ & 4791.9 & 73407 & 73001.0 & 23.69 & 1.22 & 73981 & 0.83 & 0.45 & 74165 & 74106.0 & 4.80 & 74165 & 06.7 & 9.71 & 0.202 \\
\hline & & & & 99576 & & & 2 & & 1 & .28 & & & 4.88 & & 77.4 & .07 & 0.544 \\
\hline 324 & 119445 & 0.030 & 10800.0 & 116639 & & & 2.35 & 118451 & 0.77 & 0.83 & 118771 & & 4.86 & 118771 & & 8.99 & 0.564 \\
\hline & 55397 & 0.005 & 10800.0 & & & & 1.07 & & & 0.11 & & & 4.63 & & & 9.65 & \\
\hline & & 0 & & & & & & & & & & & 4.72 & & & & \\
\hline $42 \_85$ & 61900 & 0.002 & 10800.0 & 61545 & 61266.0 & 23.81 & 0.57 & 61637 & 0.88 & 0.42 & 61746 & 61715.1 & 4.68 & 1847 & 61822.6 & 9.74 & 0.086 \\
\hline & & & & & & & 1.00 & & 0.63 & 0.86 & & & 4.54 & & & 9.82 & 0.085 \\
\hline D_1_49_90 & 56651 & 0.016 & 10800.0 & 56216 & 55813.0 & 24.15 & 0.77 & 56180 & 1.34 & 0.83 & 56321 & & 4.59 & 56532 & 56429.9 & 9.55 & 0.210 \\
\hline 324_20_1_50_90 & 59357 & 0.005 & 10800.0 & 58941 & 58577.0 & 26.03 & 0.70 & 59119 & 0.94 & 0.40 & 59253 & 59237.0 & 4.74 & 59285 & 59242.6 & 10.08 & 0.121 \\
\hline
\end{tabular}


Table 4. Results for the 818-node network

\begin{tabular}{|c|c|c|c|c|c|c|c|c|c|c|c|c|c|c|c|c|c|}
\hline \multirow[t]{2}{*}{ Problem } & \multicolumn{3}{|c|}{ CPLEX } & \multicolumn{4}{|c|}{ CGA } & \multicolumn{3}{|c|}{ MS_Heuristic } & \multicolumn{3}{|c|}{ GRASP } & \multicolumn{4}{|c|}{ CS } \\
\hline & & Gap & me & & Iverage & Time & Dev. & Solution & Time & Dev. & Best & Average 7 & & 3est & & & Dev. \\
\hline & & 0 & & & & & & & & & & 58.3 & & & & & \\
\hline & & 0 & & & & & & & & 0.06 & & & & & & & \\
\hline & & 0 & & & & 49.37 & 0.01 & 45375 & 12.09 & 0.03 & & & 6.97 & & & & 0.000 \\
\hline 3_90 & 26920 & 0 & 3004.2 & 6915 & 26901 & 48.35 & 0.02 & 26907 & 11.38 & 0.05 & 26918 & 26918.0 & 6.10 & 26920 & & .32 & 0.000 \\
\hline -90 & 28330 & 0 & 303.5 & 8320 & 28297.98 & 47.78 & 0.04 & 28309 & 8.63 & 0.07 & 28329 & 28328.6 & 6.67 & 28330 & 0.0 & 2.23 & 0.000 \\
\hline & 29680 & 0 & 780.8 & 9678 & 3.76 & 7.81 & 0.01 & 29661 & 18.28 & 0.06 & 29680 & & 6.42 & 29680 & & & 0.000 \\
\hline & 290 & 0 & & & & & 0.12 & & & 0.30 & & & 14.51 & & & & 0.000 \\
\hline & & 0 & & 3 & & & 0.09 & & & 0.11 & & & 16.90 & & & & 000 \\
\hline _95 & 90780 & 0 & 7098.1 & 90747 & 90672.9 & 84.99 & 0.04 & 90730 & 75.19 & 0.06 & 90772 & & 19.18 & 90780 & & 6.80 & 0.000 \\
\hline 85 & 4360 & 0 & 6993.7 & 4341 & 742 & 90.04 & 0.03 & 4313 & 75.05 & 0.06 & 74353 & & 16.27 & 74360 & & 6.81 & 0.000 \\
\hline & 100989 & 435. & 800.0 & 101955 & 10 & 89.19 & -0.96 & 101933 & 76.06 & -0.93 & 101996 & & 19.24 & 102000 & & 7.36 & -1 \\
\hline & & & & & & & -0.03 & 397 & & 0.01 & & & 6 & & & 4.36 & -0 \\
\hline & & & & & & & 0.10 & & & 0.13 & & & 15.93 & 55400 & & & \\
\hline & 58719 & 0.002 & 10800.0 & 58706 & 58677.58 & 87.62 & 0.02 & 58637 & 69.63 & 0.14 & 58711 & & 15.24 & & & & \\
\hline 85 & 61818 & 774.00 & 800.0 & 61839 & 61719.7 & 90.43 & -0.03 & 61814 & 71.81 & 0.01 & 61894 & & 16.16 & 61900 & & 8.71 & \\
\hline 8_90 & 53840 & 0 & 5565.0 & 53814 & 53762.18 & 87.87 & 0.05 & 53728 & 22.41 & 0.21 & 53827 & 53825.71 & 15.35 & 53840 & 39.6 & 59.56 & 0.000 \\
\hline 1_49_90 & & 0 & 5254.6 & 56605 & 56465.32 & 88.71 & 0.10 & 56602 & 34.34 & 0.10 & & 56651.61 & 16.11 & 56660 & & 42 & 0.000 \\
\hline _1_50_90 & 9360 & 0 & & 59336 & 59279.8 & 87.04 & 0.04 & 59269 & 39.84 & 0.15 & 59352 & 350.21 & 15.02 & 59360 & & 58.48 & 0.000 \\
\hline & 85876 & & 10800.0 & & & & 0.78 & & & 0.24 & 185779 & & & 185880 & & & \\
\hline 1885 & 251029 & & & & & & & & & & & & & & & & \\
\hline & 292912 & 84.45 & 10800.0 & 296763 & 296182.8 & 171.74 & -1.31 & 298217 & 757.73 & $3-1.81$ & 298547 & & 63.58 & 298582 & & & -1.936 \\
\hline 818_50_1_48_90 & 134598 & 0.001 & & 134079 & 133999.4 & & 0.39 & & 596. & 0.38 & 134474 & & 43.99 & 134598 & & 335.00 & 0.000 \\
\hline 818_50_1_49_90 & 141648 & 0.001 & 10800.0 & 140439 & 140143.4 & 174.96 & 0.85 & 141281 & 571.17 & 0.26 & 141548 & & 46.32 & 141586 & 532.8 & 356.80 & 0.044 \\
\hline 818_50_1_50_90 & 148398 & 0.001 & 10800.0 & 147202 & 147123.8 & 173.96 & 0.81 & 147931 & 667.67 & 0.31 & 148280 & 148253.14 & 46.62 & 148383 & 148321.9 & 337.4 & 0.010 \\
\hline
\end{tabular}





\section{References}

Brotcorne L., Laporte G. and Semet F. (2003). Ambulance Location and relocation models. European Journal of Operational Research, 147, pp. 451-463.

Chaves A. A. and Lorena L. A. N. (2005). Hybrid algorithms with detection of promising areas for the prize collecting traveling salesman problem. Fifth international conference on hybrid intelligent systems (HIS'05), pp. 49-54.

Chung C.H. (1986). Recent applications of the Maximal Covering Location Problem (MCLP) model. Journal of the Operational Research Society. 37, pp. 735-746.

Church R.L. and ReVelle C. (1974). Maximal covering location problem. Papers of the Regional Science Association, 32, pp. 101-118.

Corrêa F.A. and Lorena L.A.N. (2006). Using the Constructive Genetic Algorithm for Solving the Probabilistic Maximal Covering Location-Allocation Problem. I Workshop on Computational Intelligence/SBRN. Available at http://www.lac.inpe.br/ lorena/correa/Correa_Lorena_Wci_2006.pdf.

Current J. R. and O'Kelly M. (1981). Locating emergency warning sirens. Decision Sciences, 23, pp. 221-234.

Daskin M. S. (1995). Network and discrete location: models, algorithms and applications. John Wiley \& Sons, New York.

Eaton D., Hector M., Sanchez V., Latingua R. and Morgan J. (1986). Determining ambulance deployment in Santo Domingo, Dominican Republic. Journal of the Operational Research Society, 37, pp. 113-126.

Feo T. and Resende M. (1995). Greedy randomized adaptive search procedures. Journal of Global Optimization, 6, pp. 109-133.

Galvão R.D. (2004). Uncapacitated facility location problems: contributions. Pesquisa Operacional, 24: 7-38.

Galvão R. D. and ReVelle C. S. (1996). A Lagrangean heuristic for the maximal covering location problem. European Journal of Operational Research, 88, pp. 114-123.

Glover F. (1996). Tabu search and adaptive memory programming: Advances, applications and challenges. Interfaces in Computer Science and Operations Research, pp. 1-75.

Hale T. S. and Moberg C. R. (2003). Location science review. Annals of Operations Research, 123, pp. 21-35.

Hougland E. S. and Stephens N. T. (1976). Air pollulant monitor sitting by analytical techniques. Journal of the Air Pollution Control Association, 26, pp. 52-53.

ILOG CPLEX 10.0: User's Manual, France, 2006.

Larson R. C. and Odoni A. R. (1981). Urban operations research, Prentice Hall, Englewood Cliffs, N.J. 
Lorena L. A. N. and Furtado J. C. (2001). Constructive genetic algorithm for clustering problems. Evolutionary Computation, 9(3), pp. 309-327.

Lorena L. A. N. and Pereira M. A. (2002). A lagrangean/surrogate heuristic for the maximal covering location problem using Hillsman's edition, International Journal of Industrial Engineering, 9, pp. 57-67.

Marianov V. and Serra D. (1998). Probabilistic maximal covering location-allocation models for congested systems. Journal of Regional Science, 38(3): 401-424.

Marianov V. and Serra D. (2001). Hierarchical location-allocation models for congested systems. European Journal of Operational Research, 135: 195-208.

Moore G. C. and ReVelle C.S. (1982). The Hierarchical Service Location Problem, Management Science, 28 (7), pp. 775-780.

Oliveira A. C. M. and Lorena L. A. N. (2004). Detecting-promising areas by evolutionary clustering search. Bazzan, A. L. C. and Labidi, S. (Eds.) Springer Lecture Notes in Artificial Intelligence Series vol. 3171, pp. 385-394.

Oliveira A. C. M. and Lorena L. A. N. (2005). Population training heuristics. Gottlieb, J. and Raidl, G. (Eds.) Springer Lecture Notes in Computer Science Series Vol. 3448, pp. 166-176.

Oliveira A. C. M. and Lorena L. A. N. (2007). Hybrid Evolutionary Algorithms and Clustering Search. Crina Grosan, Ajith Abraham and Hisao Ishibuchi (Eds.) Springer SCI Series, vol. 75, pp. 81-102.

Pereira M. A., Lorena L. A. N. and Senne E. L. F. (2007). A column generation approach for the maximal covering location problem. International Transactions in Operations Research, v. 14, p. 349-364.

Pirkul H. and Schilling D. A. (1991). The maximal covering location problem with capacities on total workload. Management Science, 37(2), pp. 233-248.

Serra D. and Marianov V. (2004). New trends in public facility location modeling. Universitat Pompeu Fabra Economics and Business Working Paper 755. Available at http://www.econ.upf.edu/docs/papers/downloads/755.pdf. 